\title{
Optimal Collaborative Access Point Association in Wireless Networks
}

\author{
Ouldooz Baghban Karimi \\ School of Computing Science \\ Simon Fraser University \\ oba2@cs.sfu.ca
}

\author{
Jiangchuan Liu \\ School of Computing Science \\ Simon Fraser University \\ jcliu@cs.sfu.ca
}

\author{
Jennifer Rexford \\ Department of Computer Science \\ Princeton University \\ jrex@cs.princeton.edu
}

\begin{abstract}
The popularity of wireless local area networks has led to a dramatic increase in the density of access points, especially in urban areas. These access points are individually owned, placed, and power-tuned for their local users and are generally oblivious to others. On the other hand, the abundance of access points that mostly share the same upstream provider, offers opportunities for optimization of association to mitigate the negative impact of the overlapped coverage. We use this opportunity to enable collaboration by using a share of each access point's bandwidth to serve non-local users and gain access to their bandwidth in return. We extend the conventional proportional fair association through sharing and collaboration among individual networks, and present centrally optimized solutions. Our performance evaluation, based on data traces collected in $\mathbf{1 0 0}$ residential locations, demonstrate the superiority of our solution, outperforming the throughput of non-collaborative optimal access by up to $140 \%{ }^{1}$.
\end{abstract}

\section{INTRODUCTION}

The popularity of wireless local area networks for home internet access has led to a dramatic increase in the density of access points, especially in urban areas. While a user is connecting to an access point, other access points may be active within its receiving or sensing range. Unfortunately, many of these access points operate on the same channel, given the limited number of orthogonal channels in 802.11 wireless networks. This results in higher interference, higher collisions, and consequently sub-optimal throughput.

Figure 1 shows a real-world example of the received signal strength, channel usage, and rate of available access points for a representative user that we have tracked in a 25-story apartment complex. There are 54 available access points within the receiving range of the user, with $20 \%$ of them having a fairly high signal strength level as illustrated in 1(a). Figure 1(b) further illustrates the high number of active access points on each of the three orthogonal channels. Apparently, there are overlapped channels among a number of access points, known as co-channel access points [9] [3], which inevitably suffer from collisions and interference. The interference, on the other hand, depends on the usage of the channels [13], which further depends on the particular users associated to individual access points. To date, naive fixed association or simple

\footnotetext{
${ }^{1}$ Jiangchuan Liu's research is supported by a Canada NSERC Discovery Grant, an NSERC Strategic Project Grant, and a China NSFC Major Program of International Cooperation Grant (61120106008). Jennifer Rexford's research is supported by an NSF grant (1247764).
}

signal strength based association remain dominating, which in general fail to maximize the overall network throughput, not to mention ensuring fairness among the users.

The densely deployed access points, on the other hand, offer great opportunities for wireless users to smartly choose the access point to associate with. However, in home wireless networks, users can only use their dedicated access point. Fortunately, most of these networks share the same upstream internet service provider. This is an opportunity to enable collaboration and central management for optimization of access point association. In our measurements, four different providers offered residential access, two of them providing the majority of the connections. The provider can control sharing and usage limits of the collaborative networks to consistently satisfy the bandwidth requirements of local users. This way the users can take advantage of access points available to them in an area covered with multiple dense wireless networks.

Earlier studies have suggested that a proportional fair access point association ensures fairness with maximized aggregate throughput [16]. Unfortunately, this needs interference optimizations enforced during deployment. In other words, it assumes that all of the access points are working on orthogonal channels, which is simply not practical for dense networks.

In this paper, we closely examine the proportional fair access point association with densely deployed wireless networks. We enable collaboration and sharing among individual networks, and present optimized solutions for multiple access points to collaboratively serve wireless users within a set of networks that share the same upstream provider. Collaboration and bandwidth sharing is enabled by defining explicit bandwidth limits that are different for local and external users of an access point. The upstream provider can collect user signal data from individual wireless networks, centrally find the optimal association and provide it to the access points. To our knowledge, this is the first work that enables the access points to effectively collaborate with explicitly defined bandwidth limits for external users, so as to mitigate the impact of interference and node deployment in dense wireless networks. Sharing resources with external users, and interference mitigation for an inevitably user-controlled deployment, make our collaborative system fundamentally different from those of enterprise networks. Furthermore, inspired by the advances in wireless firmware virtualization [5] and traffic aggregation 


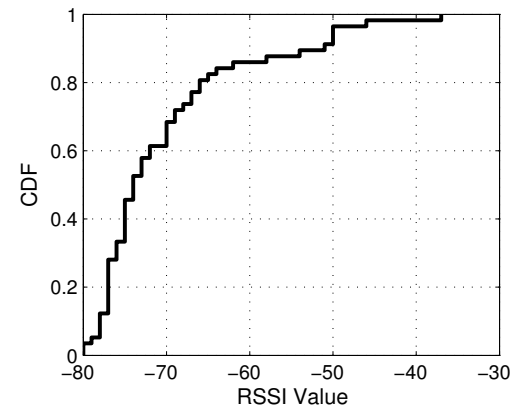

(a) Signal strengths (RSSI) of active APs

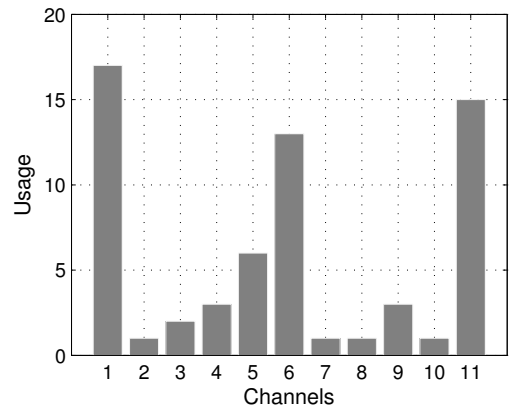

(b) Number of active APs on each channel

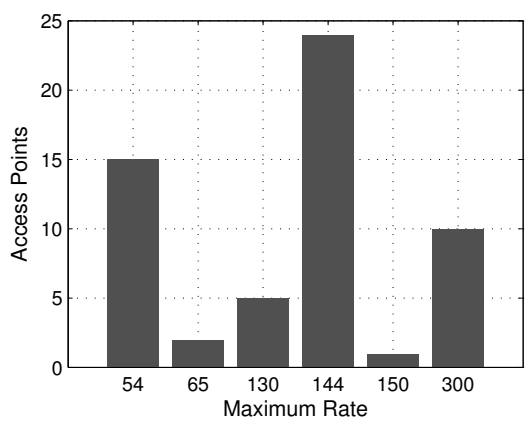

(c) Maximum rates of active APs

Fig. 1. Access points available to a sample user - from measurements in a 25-story apartment complex

[12] [8], which enable a client to connect to more than one access point, we enable concurrent association with multiple access points that fully utilizes the network resources.

Our performance evaluations, based on real data traces collected in 100 urban residential locations in Vancouver, have demonstrated the superiority of the proposed solution, which generally outperform the dedicated access point association by up to $140 \%$ in certain node deployments. We further discuss the practical implications of our solution and show that only software updates are expected at wireless clients, while only configuration changes may be required at the access points. Overall, the key contributions of this paper are as follows:

- We design a collaborative network that enables sharing access and centralized association optimization on dense deployments of individually owned access points.

- Extending proportional fair association, we model cochannel access points, sharing, and collaborative access, and provide optimal solutions in this system.

- We present measurement data from urban areas and apartment complexes in 100 locations in residential neighbourhoods to verify our design choices and show our solution can improve the overall throughput by up to $140 \%$.

The rest of this paper is organized as follows. The next section discusses the related work. We present a motivating example, and an experiment in section III. In section IV and IV-B, we present the system architecture, access model, and notations for access association optimization. We present our optimal collaborative association solution in section V. We present performance analysis of the optimization framework and evaluations in section VI. Finally, we conclude the paper and discuss future work in section VII.

\section{RELATED WORK}

In existing wireless networks, a client is either associated to a dedicated access point, or to the best access point in terms of such metrics as the received signal strength index (RSSI), potential throughput estimates [22] [17], end-to-end airtime cost [10], or a combination of different metrics defined as association cost [18]. The decision is generally made by the client locally and selfishly, which has been analyzed through game theory in different network settings, e.g., wireless access networks [24] [7] [19], cognitive radio networks [11], and cellular networks with linear topologies (linear cellular networks) [15]. It has been shown that the selfish behavior does not necessarily converge to the optimal equilibrium [23].

Optimized decentralized association algorithms [14] [23] [11] try to achieve global optimum with local client measurements. Yet certain simplifications have been made in these pioneering studies, e.g., uniform throughput among all users or linear topology, which can hardly be extended to general network settings. For example, Xu et al. [23] and Kauffman et al. [14] assume all users on a single access point have the same throughput. Hong et. al [11] limit the modelling to linear wireless networks. Our work addresses these issues.

Centralized associations, on the other hand, provide optimal associations. Koutsopoulos et al. [15] suggested that a joint access point selection and channel assignment for linear cellular networks can achieve the optimum by minimizing the maximum clique (a set of cells that all interfere with each other) load. Their solution however cannot be extended to general cellular networks, in two dimensional area since the reuse constraints are not met and the clique loads cannot be balanced. Bejerano et al. [4] proposed an association control for global max-min service in wireless networks. Zhu et al. [25] proposed a user-centric management of wireless networks to alleviate the collision problem. Liu et al. [17] also incorporated a client-AP association scheme for their practical spatial reuse antenna management solution.

Proportional fair association, among all centralized solutions, yields a good balance between system throughput and fairness. $\mathrm{Li}$ et al. [16] proposed a $2+\epsilon$ approximation of optimal proportional-fair access point association in multirate wireless local area networks. Baid et al. [3] extended the formulation to enable cooperation among the access points from different networks. The proportional fair association, however, assumes there are no two access points sharing the same channel within the same sensing range. This requirement can hardly be met in dense wireless networks and ignoring it in collaborative access will lead to a sub-optimal solution.

We extend the proportional fair association to model collaboration among the access points in a dense environment with a 


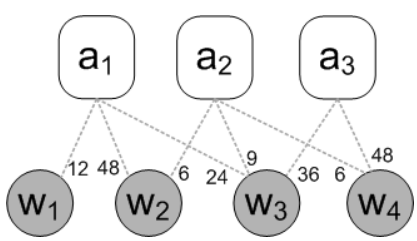

(a) Link Capacities

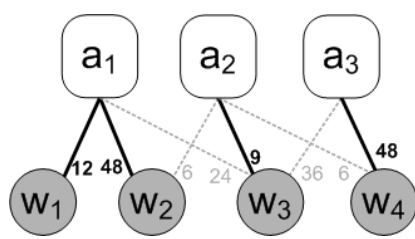

(b) Optimal Non-Collaborative

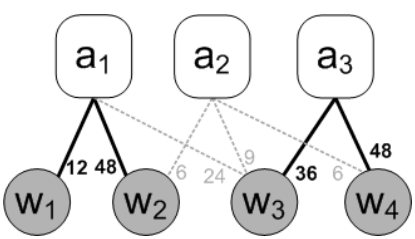

(c) Optimal Collaborative

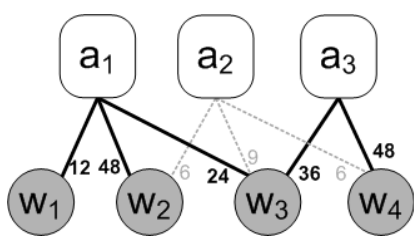

(d) Optimal Collaborative with Multiple Concurrent Associations

Fig. 2. An example Network (a) Link capacities of the sample network, (b) Optimal association decision for non-collaborative access points (dedicated access points or enterprise setting), overall throughput $=29$. (c) Optimized collaborative association using the our proposed method, overall throughput $=37$. (d) Optimized collaborative association using the proposed method when multiple associations are allowed, overall throughput $=36$.

large number of overlapped channels. We also model defining local and external bandwidth usage limits for the users, to ensure service for local users. Inspired by the advances in wireless firmware virtualization to enable a client to connect to more than one access point [5] and measurements confirming the gains of the method [21], we further extend the model to include association decisions to connect a user to multiple access points.

\section{Motivating ExAMPLE}

Consider three access points $a_{1}, a_{2}$, and $a_{3}$ and four wireless clients, $w_{1}-w_{4}$, where the rate of the wireless connection between each access point and wireless client is as indicated on the dotted lines on figure 2(a). All three access points are on the same frequency channel. Such a configuration is very common as part of a dense deployment.

Optimal non-collaborative access is illustrated in 2(b). Each user connects to its own local access point or the access point with the highest signal strength which is $w_{1}$ and $w_{2}$ to $a_{1}, w_{3}$ to $a_{2}$, and $w_{4}$ to $a_{3}$. The access points serve their users with equal serving time. The access points working on the same channel should also share the channel with equal share times. The dedicated access is also a proportional fair association. The throughput for user $w_{1}$ will be 2 . This is because it shares the access point with another user and therefore gets $1 / 2$ of serving time, and the access point shares channel with 3 cochannel access points and therefore gets $1 / 3$ of channel airtime. The rest of the users' throughputs will be 8,3 , and 16 , respectively, and the overall throughput of the network is 29 .

Optimal collaborative access, alternatively, assumes each access point allocates up to $1 / 3$ of its resources to serve external users (e.g. when $a_{3}$ serving $w_{3}$ ). As shown in figure $2(\mathrm{c}), a_{1}$ serves $w_{1}$ and $w_{2} . a_{3}$ serves $w_{3}$ and $w_{4}$, resulting in user throughputs of $3,12,6$, and 16 , respectively, and total throughput of 37 . This is the optimal collaborative access point association decision.

Optimal collaborative access with multiple associations allows multiple access point associations in collaborative association optimization, as illustrated in figure $2(\mathrm{~d}), a_{1}$ serves $w_{1}$ to $w_{3}$ and $a_{3}$ serves $w_{4}$ and $w_{3}$. In this case, the throughput for users is $2,8,4+6$, and 16 , respectively, resulting in a total throughput of 36 . The total throughput is less than the collaborative association in 2(c), however, the throughput for user $w_{3}$ is noticeably higher with all other throughputs equal to those in the network with no collaboration, illustrated in 2(b). This case is desirable when user $w_{3}$ is more important for the provider (e.g., pays more and expects higher throughput).

In addition to the increase in the system throughput, disabling the access point $a_{2}$ results in decreased interference in the network. This will improve the throughput of all users within its sensing range.

\section{System OVERVIEW}

\section{A. Architecture}

There are three different entities within collaborate networks: access points, wireless clients, and upstream providers. Collaborative access does not impose any changes on the access points. Local users of a wireless network deploy the access points. Therefore, the placement, power setting, or channel assignment of the access points have already been decided (e.g., optimized) during deployment. The deployment parameters can be changed by the users and these changes trigger association decisions at the upstream provider.

Wireless clients connect to the access points based on association decisions provided by the upstream provider. However, while initially joining the network, the wireless clients do not know the association decisions. Therefore, the association process starts by wireless client connecting to the upstream provider by the local access point or the strongest signal access point prior to having the association decision from the upstream provider. This is the initial step in association, and association between the wireless client and access point is not considered completely established. Therefore no data connections are made in this step, before the decision is announced by the upstream provider. Association decisions are made by the upstream provider and announced to the wireless clients through the initial association.

A software update at the wireless client enables the wireless client to decide the association based on the association variable announced by the upstream provider rather than the strongest signal value. It also provides firmware virtualization for multiple access point connections at the same time. The wireless clients without software updates will only be able to connect to their local access points. The upstream provider centrally optimizes the access point associations within the collaborative network. This is performed by clustering, and access point association optimization. Clustering is performed 


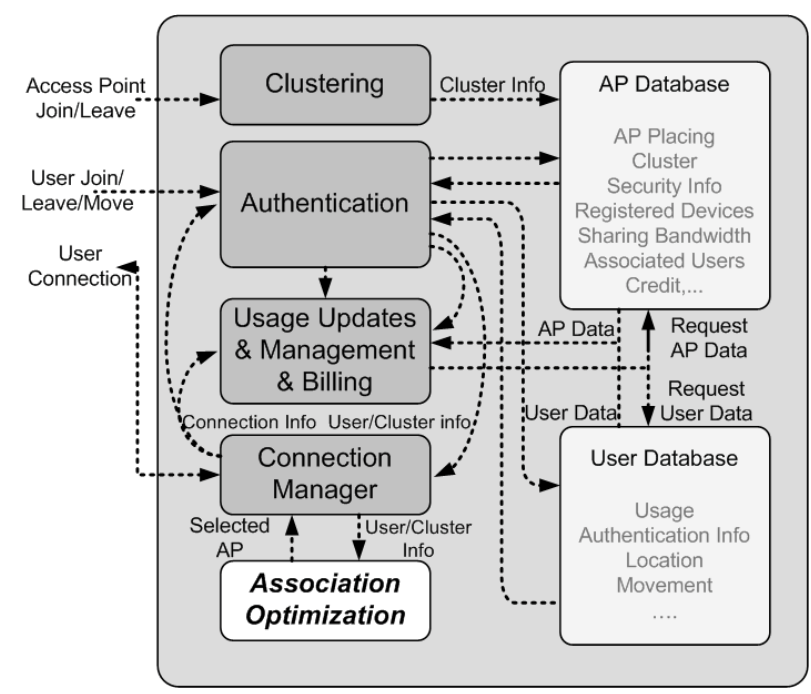

Fig. 3. AP association decision in upstream provider

on the access points and users to decide the scope of each centralized optimization. Since the wireless local area networks have a limited coverage area, the provider performs clustering based on location, i.e., access points and users within the same residential building form a cluster. This ensures the scalability of the centralized optimization by limiting the optimization variables within the scope of each cluster. Access point association is then centrally performed at the upstream provider for each cluster.

All the access points ensure secure connection to the local and external users. Security settings for each access point are performed by the local users of that access point, and shared with the upstream provider for external user access setting. This is also performed by the wireless client software update, enabling it to configure the local access point for such settings.

TABLE I

DEFINITION OF PARAMETERS

\begin{tabular}{|l|l|}
\hline Symbol & Description \\
\hline \hline$A$ & Set of all available access points \\
$m$ & Number of available access points $m=|A|$ \\
$U$ & Set of all wireless clients \\
$n$ & Number of wireless clients $n=|U|$ \\
$C$ & Set of available wireless channels \\
$c$ & Number of available wireless channels $c=|C|$ \\
\hline$p_{i}$ & Transmission power of AP $i$ \\
$R_{i}$ & Downlink rate limit of access pint $i$ \\
$L_{i}$ & Downlink usage limit of access point $i$ \\
$n_{i}$ & Number of wireless clients in network of AP $i$ \\
\hline$\alpha_{i j}$ & Max share \% of user $j$ on AP $i$ \\
$\gamma_{i j}$ & Modulation coefficient of user $j$ on AP $i$ \\
$d_{i j}$ & 1 if AP $i$ is in receiving range of user $j$ \\
$s_{i j}$ & 1 if AP $i$ is in sensing range of user $j$ \\
$w_{i j}$ & Priority of user $j$ for access point $i$ \\
$\sigma_{i j}$ & Cost of association of user $j$ to access point $i$ \\
\hline$r_{i j}^{k}$ & Link rate between AP $i$ user $j$ on channel $k$ \\
$t_{i j}^{k}$ & Air-time of AP $i$ for user $j$ on channel $k$ \\
$b_{i j}^{k}$ & Bandwidth share of user $j$ on AP $i$ on channel $k$ \\
$x_{i j}^{k}$ & Association of user $j$ to AP $i$ on channel $k$ \\
\hline
\end{tabular}

\section{B. Centralized Collaborative Access Model}

In this section, we provide the model and notations for the mathematical programming formulation of centralized access point association optimization at the upstream provider. We consider a service provider that manages $m$ wireless local area networks, each of one access point. There are $n$ wireless clients served by the same upstream provider, and the provider's goal is to maximize the total downlink throughput for all the networks, while ensuring fairness across individual users. Table I summarizes our notations.

To provide collaborative association, we assume that each access point can allocate a certain portion of its bandwidth $\alpha_{i j}$ to serve other (non-local) users to increase the global provider network throughput. $\alpha_{i j}=1$ for the local users. A user gets different share on different non-local access points based on each access point's sharing settings.

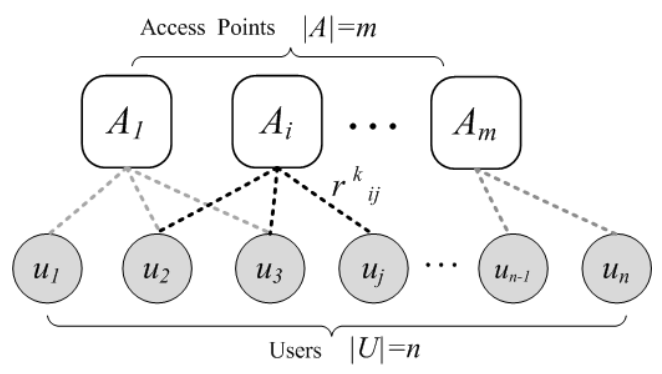

Fig. 4. Access points and wireless clients in a single provider's networks

In practice, each access point $i$ has a downlink rate limit of $R_{i}$, to provide local wireless network access to $n_{i}$ local users. This limit is based on their contract with the provider. Also, each network (access point) $i$ has a downlink usage limit $L_{i}$, also dictated by the provider. To motivate collaborative access, the provider can increase the usage limit by the usage of external users supported on each access point and decrease it by the users' usage on other networks, so that the total usage limit of a local network does not shrink for its own users.

If two access points are within each others' sensing range or have a receiving user within each others' receiving range, they should use orthogonal channels for transmission, or their simultaneous transmissions will interfere. In a densely deployed network, frequency planning can hardly guarantee orthogonal channel assignment on all access points within each other's sensing range, and careful association is therefore needed. We assume each access point on channel $k \in C$. The frequency band of the network can accommodate a total of $c$ channels. We assume multi-rate access points that serve different users with different wireless link rates. These requirements are met in most of the current access point devices. $r_{i j}^{k}$ indicates the downlink rate for user $j$ on channel $k$ of access point $i$. The $r_{i j}^{k}$ value is non-zero over only one channel if the network only uses omni-directional single input, single output access points. It can have multiple non-zero values over different channels for multiple-input multiple-output or directional antenna access points. The rates for different users 
depend on different parameters like interference, power, and modulation scheme. $t_{i j}^{k}$ denotes the fraction of time the user $j$ is served on access point $i$ on channel $k . b_{i j}^{k}$ is the bandwidth share of user $j$ on access point $i$ on channel $k\left(t_{i j}^{k} r_{i j}^{k}=b_{i j}^{k}\right)$.

The ultimate goal of the service provider is to maximize the total downlink throughput for all the networks, while ensuring fairness across individual users for customer satisfaction. Association variable $x_{i j}^{k}$ indicates the state of the association. Access point $i$ is associated to a wireless client node $j$, over channel $k$ on wireless link with capacity $r_{i j}^{k}$ if $x_{i j}^{k}$ is non-zero. We consider both single and multiple access point associations. For single access point association $x_{i j}^{k}$ is a binary variable.

\section{Collaborative Association}

The objective of wireless access network management is to optimize the $x_{i j}$ associations for maximum network utility while providing fair service to all of its users. It is known that proportional fair association provides a good balance between fairness and throughput maximization as it uses a weighted sum of logs objective function [16]. However, the proportional fair association cannot be used in local area networks where the access points only serve their local users. In this section, we provide a solution to enable sharing access to non-local users and present the optimal collaborative association with proportional fairness.

\section{A. Single Access Point Association}

We start from the single access point association, where a client can connect to only one access point, and present the access point association optimization at the upstream provider. The baseline proportional fair access on orthogonal channel access points, with no co-channel access points and no sharing at the access points, can be formulated as the following nonlinear program [16] [3]:

$$
\begin{array}{rcl}
\max & \sum_{j \in U} \log \left(\sum_{i \in A} w_{i j} x_{i j} b_{i j}\right) & \\
\text { subject to } & \sum_{i \in A} x_{i j}=1 & \forall j \in U \\
b_{i j}=\frac{r_{i j}}{\sum_{j^{\prime} \in U} x_{i j^{\prime}}} & \forall i \in A, \forall j \in U \\
x_{i j} \in\{0,1\} & \forall j \in U, \forall i \in A
\end{array}
$$

The objective is to maximize the total throughput of the network over $x_{i j}$, the association variable. In the proportional fair framework a user can connect to any access point as opposed to local wireless networks. The priority of serving a user $j$ on access point $i$ is defined by weight of the user in throughput maximization $w_{i j}$. The basic proportional fair formulation assumes the channels are all orthogonal and there are no co-channel access points. Therefore, $r_{i j}$ is used for the rate notation instead of $r_{i j}^{k}$. The first constraint dictates the client connection to only one access point; the second defines the throughput of a user based on the number of connections on the serving access point and the connection rate.
The above problem is NP-hard and Li et al. [16] have proposed a $2+\epsilon$ approximation. It relaxes the problem to a discrete linear program, and the rounding process by Shmoys and Tardos for the generalized assignment problem [20] is then applied to provide binary values of the association variable $x_{i j}$. Note that there is no collaboration among the access points. If sharing is allowed, the above solution can result in even worse throughput than the dedicated access point association, given that the channel orthogonality assumption does not hold, particularly for dense local area wireless networks.

To address this problem, we introduce access point sharing constraints to the formulation to enable collaboration with explicit sharing limits. We also add multi-channel rate specification, and frequency re-use constraints to the proportional fair association formulation to model the dense residential areas with multiple access points working on each channel. The resultant optimal collaborative access point association thus becomes:

$$
\begin{gathered}
\max \sum_{j \in U} \log \left(\sum_{i \in A} w_{i j} x_{i j}^{k} b_{i j}^{k}\right) \\
\text { subject to } \sum_{i \in A} x_{i j}^{k}=1, \forall j \in U, \forall k \in C \\
\sum_{k \in C} x_{i j}^{k}=1, \forall j \in U, \forall i \in A \\
b_{i j}^{k}=\frac{d_{i j} \alpha_{i j} r_{i j}^{k}}{\sum_{j^{\prime} \in U} x_{i j^{\prime}}^{k}} \cdot \frac{1}{\sum_{i^{\prime} \in A} s_{i^{\prime} j} \frac{\sum_{j^{\prime} \in U} \gamma_{i j} r_{i^{\prime} j^{\prime}}^{k} x_{i^{\prime} j^{\prime}}^{k}}{R_{i^{\prime}}}} \\
x_{i j}^{k} \in\{0,1\}, \forall j \in U, \forall i \in A, \forall k \in C
\end{gathered}
$$

Again, the objective is to maximize the weighted bandwidth shares of all of the users connected to an access point. The weights are computed based on association costs, as discussed in section IV. The provider whose goal is to provide highthroughput access to the customers (local network/access point owners) solves the optimization. Given a node placement, throughput is then determined by the association strategy.

The weights of the users in collaborative association are based on their contract with provider for using network resources. We define $\sigma_{i j}$, as the cost of association between user $j$ and access point $i$, to specify this priority. We define $w_{i j}=\frac{1}{1+\sigma_{i j}}$, where $\sigma_{i j}$ is calculated based on the provider pricing. The cost $\sigma_{i j}=0$ if user $j$ is the local user of the access network $i$. If user $j$ is not a local user for network $i$, the value of $\sigma_{i j}$ depends on its contract. This cost is lower for users with higher usage and bandwidth limits.

With the carrier sense multiple access (CSMA), each wireless node (access point or client) has a sensing range and a receiving range. A wireless client (end user) can sense the channel conditions of all access points within its sensing range and can associate with any access point within its receiving range. If a user is in the sensing range of an access point, the access point interferes with other wireless signals the user receives. Let $d_{i j}$ indicate if user $j$ is in the receiving range of access point $i$, and $s_{i j}$ indicate if user $j$ is in sensing range of user $i$, both of which are binary values and are available to 
the provider through feedbacks.

Since the $x_{i j}^{k}$ 's are binary values, the first constraint limits the user's connection to only one access point. The second constraint makes sure the connection of user $j$ to access point $i$ is only over a single channel. We do not need to impose any further limits on the access point channel selection. This is because each access point decides the transmission channel, and the channels used in the formulation are only for consideration of already configured co-channel access points. We will discuss the details in section V-C. The third constraint defines the throughput of user $j$ connected to access point $i$ on channel $k$. The calculation is based on the number of users to share the same access point, and the number of co-channel access points transmitting data on each others' receiving range. $d_{i j} \alpha_{i j} r_{i j}^{k}$ ensures access point $i$ is in the receiving range of user $j$ and user $j$ is within its sharing limits $\alpha_{i j}$ on access point $i . \alpha_{i j}$ is one for local users of an access point. The term, $\frac{1}{\sum_{j^{\prime} \in U} x_{i j^{\prime}}^{k}}$, takes care of time sharing among the users connected to the same access point. The term

$$
\frac{1}{\sum_{i^{\prime} \in A} s_{i^{\prime} j} \frac{\sum_{j^{\prime} \in U} \gamma_{i j} r_{i^{\prime} j^{\prime}}^{k} x_{i^{\prime} j^{\prime}}^{k}}{R_{i^{\prime}}}}
$$

ensures sharing the channel among the co-channel access points within the sensing range of user $j$ in the network. This also ensures that the association decision on the channel is based on user's perspective. i.e. the access points received or sensed by the user $j$ are not working on the same channel at the same time. $\frac{\sum_{j^{\prime} \in U} \gamma_{i j} r_{i^{\prime} j^{\prime}}^{k} x_{i^{\prime} j^{\prime}}^{k}}{R_{i^{\prime}}}$ is zero if the access point does not work on channel $k$, where the user $j$ receives on channel $k$. This term is equal to one if all the access point's users are connected to it over channel $k$. Therefore, if the working channel on access point $i^{\prime}$ is channel $k$ but there is no user connected to it, we will not consider the access point as a co-channel access point in sharing the spectrum. We will further discuss this in section V-C. Modulation $\gamma_{i j}$ indicates the modulation coefficient for user $j$ on access point $i$. The modulation schemes and transmission rates are decided by the MAC layer. For any association combination in the system

$$
\sum_{k \in C} \sum_{j \in U} t^{i j} r_{i j}^{k} \gamma_{i j}=R_{i}
$$

holds for all access points.

The nonlinear optimization problem (2) can be relaxed to a discretized linear program without the integrality constraint on $x_{i j}^{k}$ to allow multiple associations. We can then apply rounding techniques to obtain the single access point association.

\section{B. Multiple Access Point Association}

In practice, an access point may not be willing to provide more than a certain amount of its available bandwidth (indicated by $\alpha_{i}$ 's in the optimization) to a certain user. If the user demands even higher throughput, we suggest that it can connect to multiple access points simultaneously (see Figure 2(d)). Association to multiple access points is possible by time sharing among the connections in the user [5].

This enables the provider to keep the connections for the user, which could use multiple TCP or TCP-friendly rate controlled connections [6] on the multiple access points. The challenge, however, is to increase its throughput without increasing the interference or contention level in the network.

To this end, we model the multiple access point association using convex optimization, as below.

$$
\begin{aligned}
& \max \sum_{j \in U} \log \left(\sum_{i \in A}\left(w_{i j} d_{i j} \sum_{k \in C} t_{i j}^{k} r_{i j}^{k}\right)\right) \\
& \text { s.t. } \quad \sum_{i \in A} t_{i j}^{k} \leq 1 \quad \forall j \in U, \forall k \in C \\
& \sum_{j \in U}\left(t_{i j}^{k}+\sum_{i^{\prime} \in A} s_{i^{\prime} j} \sum_{j^{\prime} \in U} t_{i^{\prime} j^{\prime}}^{k}\right) \leq 1 \quad \forall i \in A, \forall k \in C \\
& \sum_{k \in C} t_{i j}^{k} \leq \alpha_{i j} \quad \forall i \in A, \forall j \in U \\
& 0 \leq t_{i j}^{k} \leq 1, \forall k \in C \quad \forall j \in U, \forall i \in A
\end{aligned}
$$

The first constraint limits the total allocated time fraction of each user $j$ over a channel $k$ to 1 . The second constraint says that the fraction of time a user $j$ is connected (to any access point) cannot be more than 1 . It also ensures the time sharing of co-channel access points within the sensing range of user $j$ over channel $k$. The third constraint says that a user $j$ 's fraction of time being served on access point $i$ over all channels cannot be more than $\alpha_{i j}$ of the access point's air-time.

The convex optimization does not determine the number of associations. It may assign a serving time over all access points in the receiving region of a user. Hence, we limit the maximum number of simultaneous access points associations for a user to $c$, the number of channels. This is because of the limitation on the frequency channels available for each wireless access point. If a wireless client is connected to more than $c$ access points at a time, the $(c+1) t h$ access point channel will interfere at least with one of the current associations and therefore the connection will not work at the desired rate. The number is different for directional antennas.

The provider can solve the convex program in polynomial time. Then, it applies rounding to omit the associations with connection rates lower than $\beta$ times of the link rate to have at most $c$ connections at each access point by setting the air-time share of the rest of the users to zero. Assume $t_{i j}^{\prime k}$ denotes the transmission time shares after dropping extra connections. We compute the new bandwidth and the final association variable and on each client:

$$
\begin{aligned}
b_{j}^{\prime}=\sum_{i \in A} \sum_{k \in C} r_{i j}^{k} t_{i j}^{\prime k} d_{i j} \quad \forall j \in U \\
x_{i j}^{\prime k}=\frac{r_{i j}^{k} t_{i j}^{\prime k}}{b_{j}^{\prime}} \quad \forall j \in U, \forall i \in A, \forall k \in C
\end{aligned}
$$

We recompute the new transmission times using the new association variables $x_{i j}^{\prime k}$ and the ratio of the used bandwidth 


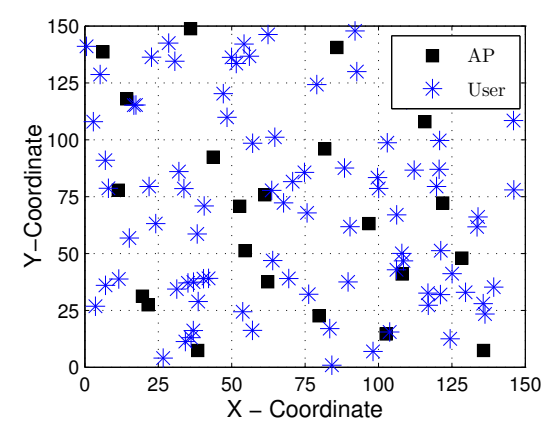

(a) Placement of APs and Users

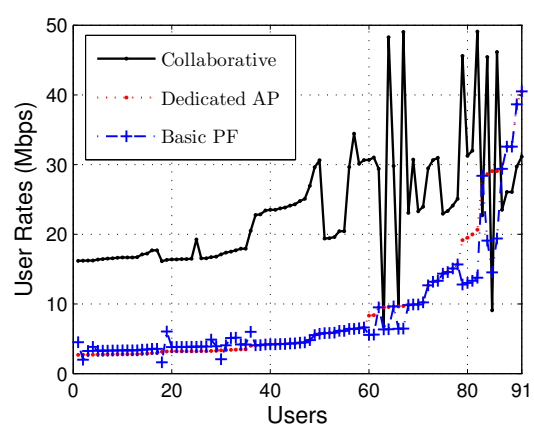

(b) Wireless Client Rates

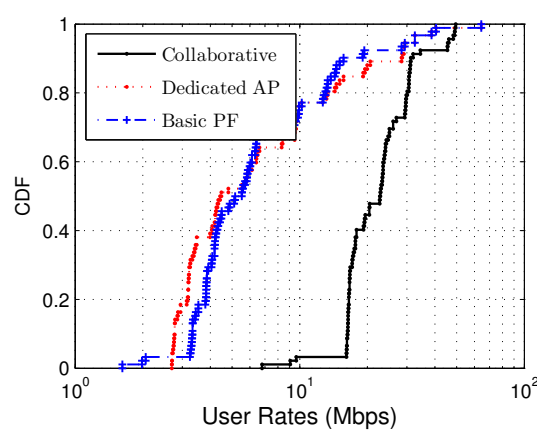

(c) $\mathrm{CDF}$ of Client Rates

Fig. 5. Client rates for wireless networks with 15 access points and 91 client users in a $150 \mathrm{~m} \times 150 \mathrm{~m}$ area

$b_{j}^{\prime} / r_{i j}^{k}$. Then, we round up the non-zero association variables to 1 and recompute the air-time share. The approximation factor for the rounding algorithm is $\frac{\beta^{2}}{c}$. The provider can also use the convex optimization (3) and the rounding algorithm above for wireless clients connecting only to one access point. It provides a looser approximation factor than that of the discrete approximation for non-linear program (2). Due to convexity of the optimization equation, it is fast enough even for large networks.

As mentioned earlier, the provider sets the $R_{i}, L_{i}$, and $\sigma_{i j}$ values for the access points, based on service contracts. The provider also announces the $x_{i j}^{k}$ 's to the access points.

\section{Channel Assignment}

We use multi-channel formulation in the collaborative access point association formulation. However, the channel in the optimization formulation is used for the one or multiple channels that the MAC layer has already selected for transmission and have provided the rates over it. In other words, the channels selected for transmission from the MAC layer are used to formulate the multi-channel collaborative access point association. Therefore, the $r_{i j}^{k}$ values are provided on the channels that the access point has already selected and decided the modulation scheme for, and the $r_{i j}^{k}$ values over the rest of the channels are zero.

We assume that the conventional channel selection scheme is used in the MAC layer, where each access point selects the least contended orthogonal channel in the channel re-use map of the network by sensing the other access points in the vicinity. If the access points are single input single output, as in $802.11 \mathrm{a} / \mathrm{b} / \mathrm{g}$, they can only have non-zero $r_{i j}^{k}$ values for one $k \in C$. If the access points are multiple input multiple output, as in $802.11 \mathrm{n}$, they can have non-zero $r_{i j}^{k}$ values over $c$ different channels. The term

$$
\frac{\sum_{j^{\prime} \in U} \gamma_{i j} r_{i^{\prime} j^{\prime}}^{k} x_{i^{\prime} j^{\prime}}^{k}}{R_{i^{\prime}}}
$$

only takes 0 or 1 values for single input single output access points. It can have values between 0 and 1 for multiple input multiple output access points that sum up to 1 over all channels for a single user. However, connection to a user over a single channel is the common choice based on the limited number of orthogonal channels.

The access points also set the values of $d_{i j}, s_{i j}$, and $\gamma_{i j}$ after sensing the access points in their vicinity and report these values along with the user feedback on $r_{i j}^{k}$ values from the user to the provider for the optimization.

The provider can compute the optimal values of the nonlocal $\alpha_{i j}$ and recommend these values to the wireless clients to configure the access points locally according to these values. In this paper, we do not consider details of setting $\alpha_{i j}$ values and consider them as predefined values.

\section{PERFormance EVAluAtion}

\section{A. Data Collections}

To accurately emulate real-world WiFi deployment, we have collected residential $\mathrm{WiFi}$ data in more than 100 locations in different residential locations in Vancouver, Canada. We used an open source Wi-Fi scanning software, InSSIDer, and WiFiExplorer for scanning the wireless access points and their signal strength in these locations. For each location, we have collected MAC address, SSID, BSSID, modem vendor, network type, operation mode, RSSI value, maximum, average, and minimum signal values and percentages, security type, encryption, band, channel, signal to noise ratio (SNR), minimum, maximum and average noise values and percentages, maximum rate, and stability in RSSI values in five-minute time intervals at different times of a day. We used the collected data in setting up the evaluation scenarios in our numerical analysis and simulations. Our dataset is available online.

Our collected data suggested that in many residential buildings there are more than a handful of access points that share the same provider, and have strong enough signal to be considered for association for a single wireless user. We also observed that home sharing devices (e.g., gaming consoles, phones with WiFi tethering), although working in the $\mathrm{WiFi}$ frequency, usually use lower transmission powers and are usually not discoverable by the devices more than a few meters away. Therefore, the devices using WiFi for home sharing can continue working within the provided scheme.

We also collected application and traffic measurements in a few residential buildings. We observed that downlink trans- 


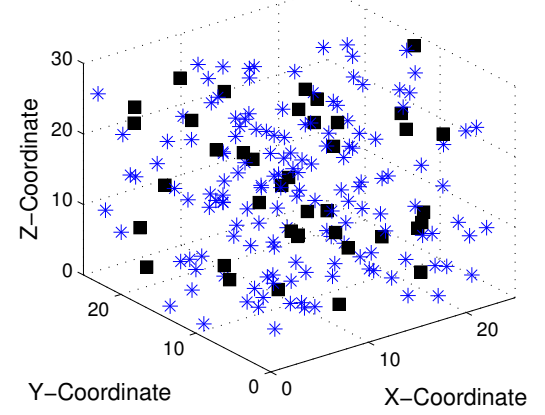

(a) Placement of APs and Users

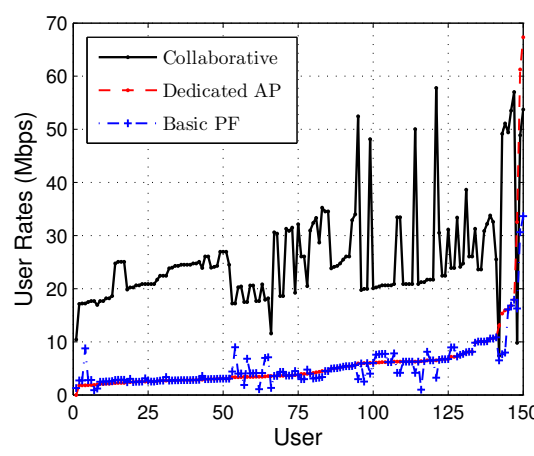

(b) Wireless Client Rates

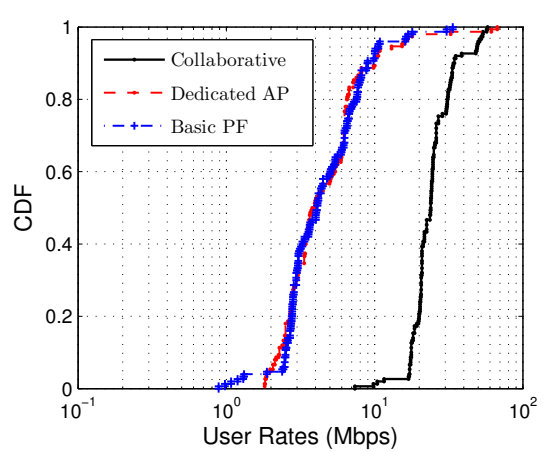

(c) CDF of Client Rates

Fig. 6. Client rates for wireless networks in a residential building of $25 m \times 25 m \times 30 m$ area: 10 floors with 4 access points on each floor

missions from the access points account for the majority of the transmission in residential wireless networks. We also found out that the wireless capacity, reduced by high contention and suboptimal associations, is the bottleneck in providing a high throughput internet downlink for applications like streaming in high density areas. This could be solved by controlled sharing and collaborative access.

\section{B. Simulations}

1) System Configuration: We used CVX [1], a package for specifying and solving convex programs, to solve the the convex problem in multiple access point connection and lpsolve to solve the discrete LP problem.

We set all the access points to work on the $2.4 \mathrm{GHz}$ band on 11 channels of $802.11 \mathrm{~b} / \mathrm{g} / \mathrm{n}$. The default transmission power level for each node in the network is $10 \mathrm{dBm}$, with a sensing range of $125 \mathrm{~m}$ and a receiving range of $35 \mathrm{~m}$ for indoor access points. The interference coefficient for completely overlapping channels is one, and the coefficients for one, two, three, four, and five channel separations are 0.7906, 0.5976, 0.2651, 0.00627 , and 0.0012 , respectively [2]. There is no interference between orthogonal channels.

Our proposed system solves a problem which is more evident for complex systems with a large number of access points at each location. We simulate this environment using our measurements from residential networks. We deployed the access points and wireless clients with clustered distributions and in 2D and 3D scenarios, respectively modelling flat residential areas and residential high-rises. In the 2D scenario, we divided the given area into smaller, non-overlapping squareshaped areas and randomly placed an access point within the borders of each small area. We then placed a random number of users (up to $n_{\max }$, the maximum number of local users for each access point) for each access point within the area to resemble a residential wireless local area network.

For 3D configurations, we set the height of each floor to $3 \mathrm{~m}$, and the above 2D placement is then applied for each floor.

2) Evaluation Results and Analysis: For comparison, we also implemented dedicated access point association (default access in residential wireless networks), and the basic proportional fair access point association [16]. We enabled a share portion of $\alpha$ on each access point, for the proportional fair association as well. Therefore, the bandwidth sharing available for proportional fair will be the same as for the collaborative access. However, proportional fair does not consider cochannel access points in making association decisions.

We sorted the user rates in the figures based on the client rates in the dedicated access. The collaborative access rates and basic proportional fair association access rates of the users are shown with the same order to illustrate the gains of each client using different associations.

Figure 5(b) illustrates the user rates in a sample configuration of a wireless networks in a $150 \mathrm{~m} \times 150 \mathrm{~m}$ area. The access point and the user placement are depicted in Figure 5(a), where all the access points are one meters above the ground. $\alpha_{i j}$ is $30 \%$ for all of the foreign users on an access point $i$ and $100 \%$ for all of its local users. Each access point has at most 6 local users. The cost of association is considered to be $\sigma_{i j}=0$ for local users, and 0.5 for other users. In this network, we assumed $R_{i}$, the downlink rate limit from the provider, is equal to the maximum achievable wireless rate at the access point. This assumption implies that the bottleneck is the wireless link capacity.

Figure 6(b) shows the user rates in a 3D scenario, which consists of 10 floors, each of $25 \mathrm{~m} \times 25 \mathrm{~m}$. The access point and user placement is depicted in Figure 6(a). We have deployed four access points in each floor and each access point has at most six users connected. The sharing parameter is $\alpha_{i j}=$ $30 \%$ for non-local users. Because of the high number of nodes in this configuration, we have used the convex optimization for this scenario. The number of multiple connections allowed is three, but most of the users connect to only one access point. We can see that the higher the number of access points and users per access point, the better the collaborative access performs in comparison to dedicated access point users.

The average wireless client rate in this scenario is improved by about $140 \%$. This increase is due to three main reasons: (a) The collaborative association uses fewer access points, and therefore fewer collisions occur for co-channel access points, resulting in less interference for non-orthogonal channel users; (b) Majority of the users are connected to the access points providing higher rates; (c) The load is better balanced across 

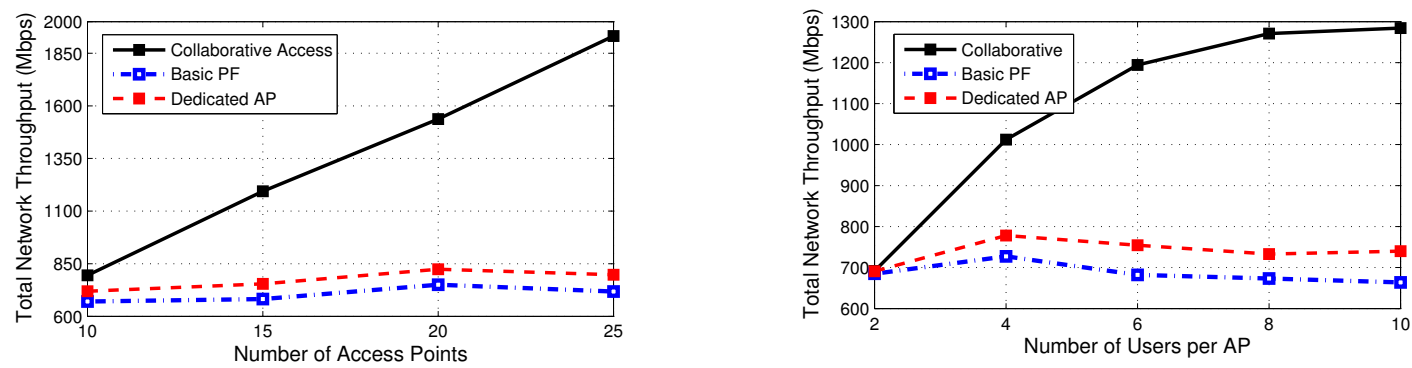

Fig. 7. Impact of (a) the number of access points, and (b) the number of users per access points on the total network throughput

access points, instead of constrained to serving local users. We also find that the highest increase is for low-rate users, due to elimination of co-channel access points.

Figures 7(a) and 7(b) illustrate the impact of the number of deployed access points and number of users per access point on the collaborative access throughput. As illustrated in these figures, availability of more access points with different rates and connection qualities to choose from in creating the associations, and possibility of turning off the unused access points help the solution gracefully adapt to increase in the deployment and demand. The gain is the most for 6-12 users per access point, ideal for residential wireless access control.

\section{CONCLUSION AND FUtURE WORK}

With wireless local area networks being the main end-point access method in homes and offices, we are expecting higher densities in access point deployments in the next couple of years. The high deployment density, given there is no collaboration among these networks, increases interference and co-channel access points. This results in reduced throughpu.

We studied the challenges in achieving high-throughput wireless access in dense wireless networks. We addressed effective access point association to maximize user throughput. Our overall solution is a centralized optimization based on a proportional fair access point association mathematical formulation. It is solved at the common upstream provider of the local networks, to maximize the provider network throughput as well as the experience for individual end users. Our simulation results confirm the effectiveness of the proposed solution with considerable throughput gains.

\section{REFERENCES}

[1] CVX: Matlab software for disciplined convex programming, version 1.21. http://cvxr.com/cvx/, Apr. 2011.

[2] Angelakis, V. andTraganitis, A., And Siris, V. Adjacent channel interference in a multi-radio wireless mesh node with $802.11 \mathrm{a} / \mathrm{g}$ interfaces. In IEEE INFOCOM (2004).

[3] Baid, A., Schapira, M., Seskar, I., Rexford, J., And RayCHAUDHURI, D. Network cooperation for client-AP association optimization. In International Workshop on Resource Allocation and Cooperation in Wireless Networks (2012).

[4] Bejerano, Y., HAN, S.-J., AND LI, L. E. Fairness and load balancing in wireless LANs using association control. In ACM MobiCom (2004), pp. 315-329.

[5] Chandra, R., Bahl, P., And Bahl, P. MultiNet: connecting to multiple ieee 802.11 networks using a single wireless card. In IEEE INFOCOM (2004), vol. 2, pp. $882-893$.

[6] ChEn, M., AND ZAKHOR, A. Multiple TFRC connections based rate control for wireless networks. IEEE Transactions on Multimedia 8, 5 (2006), $1045-1062$.
[7] Ge, W., Ji, H., Leung, V., And Si, P. Access point selection for WLANs with cognitive radio: A restless bandit approach. In IEEE International Conference on Communications (2011), pp. 1-5.

[8] Giustiniano, D., Goma, E., Lopez Toledo, A., Dangerfield, I., MORILLO, J., AND RODRIGUEZ, P. Fair WLAN backhaul aggregation. In MobiCom (2010), pp. 269-280.

[9] GosLing, W. A simple mathematical model of co-channel and adjacent channel interference in land mobile radio. IEEE Transactions on Vehicular Technology 29, 4 (1980), 361 - 364

[10] He, Y., Perkins, D., AND Velaga, S. Design and implementation of CLASS: A cross-layer association scheme for wireless mesh networks. In IEEE INFOCOM (2010), pp. 1-6.

[11] Hong, M., Garcia, A., AND BARRERA, J. Joint distributed access point selection and power allocation in cognitive radio networks. In IEEE INFOCOM (2011), pp. 2516-2524.

[12] Kandula, S., Lin, K. C.-J., Badirkhanli, T., AND Katabi, D. FatVAP: Aggregating AP backhaul capacity to maximize throughput. In USENIX NSDI (2008), pp. 89-104.

[13] KARIMI, O. B., LiU, J., AND LI, Z. Multicast in multi-channel wireless mesh networkss. In IFIP Networking (2010), pp. 148-159.

[14] Kauffmann, B., Baccelli, F., Chaintreau, A., Mhatre, V., PaPAGIANNAKI, K., AND DIOT, C. Measurement-based self organization of interfering 802.11 wireless access networks. In IEEE INFOCOM (2007), pp. 1451-1459.

[15] Koutsopoulos, I., AND TAssiulas, L. Joint optimal access point selection and channel assignment in wireless networks. IEEE/ACM Transactions on Networking 15, 3 (2007), 521-532.

[16] Li, L., PAL, M., AND YAng, Y. Proportional fairness in multi-rate wireless LANs. In IEEE INFOCOM (2008), pp. $1004-1012$.

[17] LiU, X., Sheth, A., Kaminsky, M., Papagiannaki, K., Seshan, S., AND STEEnKISTE, P. Pushing the envelope of indoor wireless spatial reuse using directional access points and clients. In ACM MobiCom (2010), pp. 209-220.

[18] Musacchio, J., AND Walrand, J. Wifi access point pricing as a dynamic game. IEEE/ACM Transactions on Networking 14, 2 (2006), 289-301.

[19] Shak Kottai, S., Altman, E., And Kumar, A. Multihoming of users to access points in WLANs: A population game perspective. IEEE Journal on Selected Areas in Communications 25, 6 (2007), 1207-1215.

[20] Shmoys, D. B., AND TARdos, E. An approximation algorithm for the generalized assignment problem. Mathematical Programming 62 (1993), 461-474.

[21] Soroush, H., Gilbert, P., Banerjee, N., Levine, B. N., Corner, M., AND COX, L. Concurrent wi-fi for mobile users: analysis and measurements. In ACM CoNEXT (2011), ACM, pp. 1-12.

[22] Sun, T., ZhANG, Y., AND TRAPPE, W. Improving access point association protocols through channel utilization and adaptive switching. In IEEE Conference on Mobile Adhoc and Sensor Systems (2011), pp. $155-157$.

[23] Xu, F., Tan, C., LI, Q., Yan, G., AND Wu, J. Designing a practical access point association protocol. In IEEE INFOCOM (2010), pp. 1-9.

[24] Xu, W., HuA, C., AND HuANG, A. Channel assignment and user association game in dense 802.11 wireless networks. In IEEE International Conference on Communications (2011), pp. 1-5.

[25] ZHU, Y., MA, Q., Bisdikian, C., AND YinG, C. User-centric management of wireless LANs. IEEE Transactions on Network and Service Management 8, 3 (2011), $165-175$. 